\title{
特別講演 3 \\ Pediatric asthma in Sweden. Changes in management, hospitalization rates and mortality.
}

\author{
Göran Wennergren \\ Department of Pediatrics, Göteborg University, Queen Silvia Children's Hospital
}

\section{Changes in asthma management and hospitalization rates}

During the last two decades we have seen drastic changes in the management of pediatric asthma, in asthma hospitalization rates and in asthma mortality. For example, in Göteborg, Sweden, there has been a continuous decrease in number of hospital days, admissions and individual patients admitted due to asthma in the age group 5-18 years. The number of hospital days is now only $5 \%$ of what it was in 1985. Among children in this age group we see a strong correlation between decreased number of hospitalizations for acute asthma and treatment with inhaled corticosteroids. However, we have not seen a corresponding decrease in hospitalizations in young children, neither in the age group 2-4 years, nor in hospitalizations for asthmatic symptoms/wheezing bronchitis in children below two years of age. In the young age group increased use of inhaled corticosteroids has not been reflected by an overall decrease in hospitalizations. Effective treatment also for the young age group constitutes a major therapeutic challenge. The decrease in asthma hospitalizations among school children represents a great success in pediatric allergy. The most drastic decrease was seen when inhaled corticosteroids became commonly used, but interestingly, the decrease has continued also in recent years. Better overall asthma control is probably also the result of improved patient education and possibly, lately, introduction of other anti-inflammatory drugs, i.e. the leukotriene antagonists.

\section{Mortality in asthma}

Despite an increased prevalence of the disease, the mortality in asthma has decreased during the last 15-20 years. In Sweden the asthma mortality in children and young adults has decreased from 5 per million/year in the mid-1980s to 0.5 per million/year today. This corresponds to 1-3 deaths per year. In Sweden children with asthma are mainly cared for by pediatricians and pediatric allergists and not by general practitioners. This has been suggested to be one explanation for our relatively low asthma hospitalization and mortality rates, compared to some other European countries. In addition, pediatricians and pediatric allergists train the GP colleagues in management of pediatric asthma. The main preventable factors seem to be undertreatment, non-compliance with anti-inflammatory treatment, and, in young adults, also psychosocial factors including alcohol or drug abuse. Notably many of the deaths in an acute asthma attack have been caused by food allergy. Especially, fatal cases of acute asthma attacks triggered by food allergy to peanuts, soya-beans or hazel-nuts have received attention. 
In some of the fatal cases there has been an underestimation of the severity of the asthma and lacking awareness of the risks of the disease. Increased knowledge and awareness of the risk factors for death in asthma are needed to prevent these tragic cases.

\section{References}

Wennergren G, Kristjánsson S, Strannegård I-L. Decrease in hospitalization for treatment of childhood asthma with increased use of antiinflammatory treatment, despite an increase in the prevalence of asthma. J Allergy Clin Immunol 1996; 97: 742-8.

Wennergren G, Strannegård I-L. Asthma hospitalizations continue to decrease in schoolchildren but hospitalization rates for wheezing illnesses remain high in young children. Acta Paediatr 2002; 91: 1239-45.

Formgren H, Bergström SE, Boman G, Foucard T, Hedlin G, Hörte LG, Spetz-Nyström U. Can unexpected deaths in asthma be prevented? Journal of the Swedish Medical Association 2001; 98: 5314-21. 